\title{
Gene network of type 2 diabetes: reconstruction and analysis
}

\author{
Zamyatin Vladimir \\ ICG SB RAS, Novosibirsk, Russia \\ NSU, Novosibirsk, Russia \\ zamyatin@bionet.nsc.ru \\ Afonnikov Dmitry \\ ICG SB RAS, Novosibirsk, Russia \\ NSU, Novosibirsk, Russia \\ ada@bionet.nsc.ru
}

\author{
Mustafin Zakhar \\ ICG SB RAS, Novosibirsk, Russia \\ NSU, Novosibirsk, Russia \\ mustafin@bionet.nsc.ru \\ Klimontov Vadim \\ ICG SB RAS, Novosibirsk, Russia \\ NSU, Novosibirsk, Russia \\ klimontov@mail.ru
}

\author{
Matushkin Yury \\ ICG SB RAS, Novosibirsk, Russia \\ NSU, Novosibirsk, Russia \\ mat@bionet.nsc.ru \\ Lashin Sergey \\ ICG SB RAS, Novosibirsk, Russia \\ NSU, Novosibirsk, Russia \\ lashin@bionet.nsc.ru
}

\begin{abstract}
Currently, due to the appearance of an increasing number of genomic, molecular, histological data, there is an intensive detailing of individual molecular genetic systems of the human body and phenotypic deviations caused by violations in one or more elements of these systems. At the same time, there is no, as such, a holistic understanding of the nature of the formation and course of type II diabetes, which includes an enough available experimental data. In this study, we reconstructed gene networks of transcriptional regulation, functional connectivity, and protein-protein interactions for type 2 diabetes. Information on the evolutionary age of genes was superimposed on the network; it was shown that the genes in question are predominantly "evolutionarily old". New genes have been found that were not previously associated with type 2 diabetes, such as PER1, PER3, ARHGEF4, genes whose homologues are associated with the onset of diabetes in mice CLOCK, ARNTL (encoding transcription factors) - the goals for subsequent experimental confirmation. Validation of gene networks by analysis of tissue-specific expression has shown that most genes included in putative signal transduction pathways are expressed in the same tissues.
\end{abstract}

Keywords - gene networks, type 2 diabetes, SNP

Motivation and aim

\section{Motivation}

Type 2 diabetes is a multifactorial disease caused by a combination of genetic and environmental factors. Today, systems biology approaches, and in particular, network approaches, are powerful tools for studying multifactorial diseases. They are based on knowledge of the physical or functional interactions between molecules, usually presented as a network of bonds. Such networks not only report connections between individual nodes, but also implicitly organize higher-level cellular connections. In this paper, we consider networks of transcriptional regulation, proteinprotein interactions, functional connectivity, bringing them to the general value of gene networks - molecular genetic systems, which ensure the development of phenotypic properties of organisms based on genetic information.

Aim - reconstruction and analysis of the gene network of type 2 diabetes mellitus.

\section{Methods}

Based on the analysis of literature data, as well as a number of databases, a sample of genes associated with type 2 diabetes was formed. Among them, transcription factors (TFs) were identified, and using the Hocomoco database [1], TF binding sites were searched in 5, non-coding gene regions from the obtained samples. Using the Orthoscape program [2], information was obtained on the philostratigraphic age and divergence index for each gene.
Intrapopulation variability was estimated using SNP data from the project "1000 human genomes" [3]. Gene networks were reconstructed in the Cytoscape program [4] based on data on interactions extracted from GeneMANIA [5] and StringDB [6].

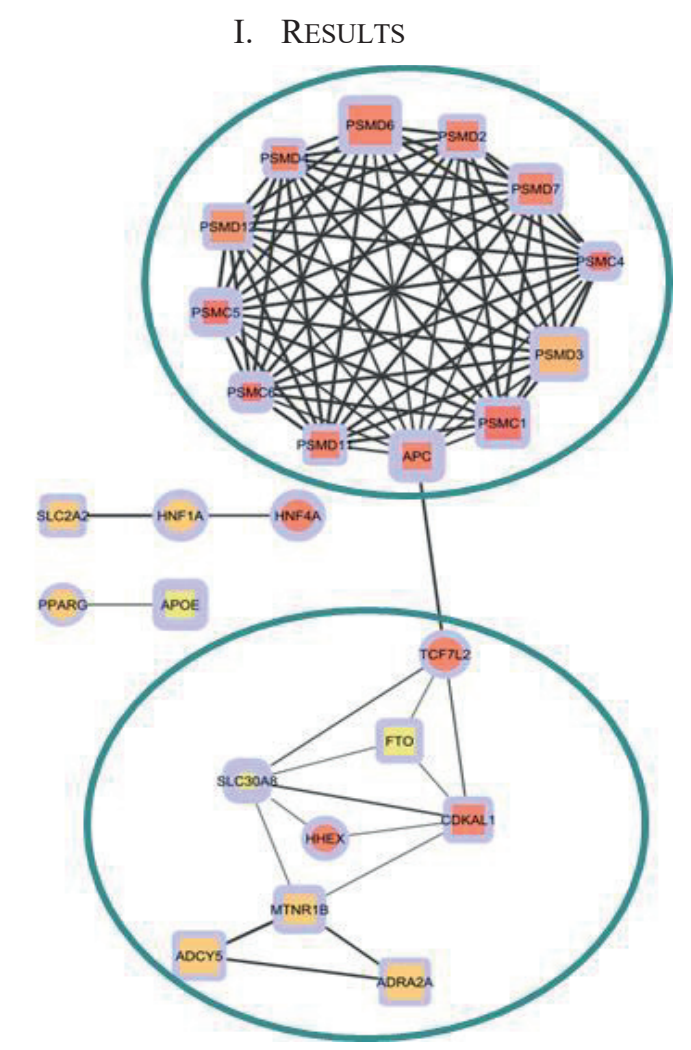

Evolutionary network map of functional connectivity. The bulk of the network genes, based on our calculations, is rather "evolutionarily old". Two circuits are distinguished - signal transmission paths

After applying the previously obtained information, an analysis of gene networks was carried out. Regulatory circuits were identified, the violations in the elements of which can lead to disease. They contain new genes not previously associated with $\mathrm{T} 2 \mathrm{D}$, such as PER1, PER3, $A R H G E F 4$, genes whose homologues are associated with the onset of diabetes in mice - CLOCK, ARNTL (encoding transcription factors) [7], as well as genes that are not in the original sample but associated with $\mathrm{T} 2 \mathrm{D}$, such as $P D X 1$, which validates our chosen methodology.

The gene network analysis is an effective tool for a comprehensive theoretical study of human diseases and can be used not only to search for and prioritize genes associated 
with diseases, but also to explain the molecular genetic mechanisms behind them.

\section{ACKNOWLEDGMENT}

Supported by the RFBR (20-04-00885).

\section{REFERENCES}

[1] Kulakovskiy IV, Vorontsov IE, Yevshin IS, Soboleva AV, Kasianov AS, Ashoor H, Ba-Alawi W, Bajic VB, Medvedeva YA, Kolpakov FA, Makeev VJ. HOCOMOCO: expansion and enhancement of the collection of transcription factor binding sites models. Nucleic Acids Research. 2015; 44 (D1): D116-25. doi: 10.1093/nar/gkv1249.

[2] Mustafin, ZS, Lashin, SA, Matushkin, YG, Gunbin, KV, Afonnikov, DA. Orthoscape: a cytoscape application for grouping and visualization KEGG based gene networks by taxonomy and homology principles. BMC Bioinformatics. 2017; S1(18): 1-9. doi: 10.1186/s12859-016-1427-5
[3] Auton, Adam, Abecasis, Gonçalo R., Altshuler, David M. Durbin, Richard $\mathrm{M}$ et al. A global reference for human genetic variation // Nature. 2015. doi: 10.1038/nature15393

[4] Shannon P, Markiel A, Ozier O, Baliga NS, Wang JT, Ramage D, Amin N, Schwikowski B; Ideker T. Cytoscape: A Software Environment for Integrated Models of Biomolecular Interaction Networks. Genome Research. 2003; 11(13): 2498-2504. doi: 10.1101/gr.1239303

[5] Szklarczyk D, Franceschini A, Wyder S, Forslund K et al. STRING v10: protein-protein interaction networks, integrated over the tree of life. Nucleic Acids Research. 2014; D1 (43). doi: 10.1093/nar/gku1003

[6] Warde-Farley D, Donaldson S, Comes O, Zuberi K et al. The GeneMANIA prediction server: biological network integration for gene prioritization and predicting gene function. Nucleic Acids Research. 2010; S2 (38). doi: 10.1093/nar/gkq537

[7] Marcheva B Ramsey K, Buhr E, Kobayashi Y, et al. Disruption of the clock components CLOCK and BMAL1 leads to hypoinsulinaemia and diabetes. Nature (2010). doi:10.1038/nature09253 